\title{
Conceptions and Modeling for Transmitted Information Evaluation by ANN
}

\author{
Algis GARLIAUSKAS \\ Vilnius Gediminas Technical University \& Institute of Mathematics and Informatics \\ Saulètekio 11, LT-2054, Vilnius, Lithuania \\ e-mail: galgis_1@ktl.mii.lt
}

Received: September 2004

\begin{abstract}
In this paper, the main measure, an amount of information, of the information theory is analyzed and corrected. The three conceptions of the theory on the microstate, dissipation pathways, and self-organization levels with a tight connection to the statistical physics are discussed. The concepts of restricted information were introduced as well as the proof of uniqueness of the entropy function, when the probabilities are rational numbers, is presented.

The artificial neural network (ANN) model for mapping the evaluation of transmitted information has been designed and experimentally approbated in the biological area.
\end{abstract}

Key words: information theory, entropy, amount of information, artificial neural networks.

\section{Introduction}

The information theory as a new branch of technical systems and probability theory in mathematics possessed extensive potential applications to the transmitted systems of information or communication systems. Like several branches of mathematics, the information theory is of physical origin. The information may be sending of a message by phone, radio, telegraph, visual communication means, or any other ones by which information is conveyed from a transmitter to the receiver through channels of the transmission network.

The information theory was founded on the conception of physical entropy, introduced by Boltzmann (1968), that explains the behavior of thermodynamical equilibrium. First, the entropy means of evaluating information was suggested by Hartley (1928). However, the theoretical background of information theory was principally originated by Claude Shannon $(1948,1949)$, based upon two outstanding cotributions to the mathematical theory of communication. Later on, over thousand research contributions were published both as an attempt of generalizing the theory and applying in technical systems.

The main measure in the information theory is the amount of information. The first attempt to define this measure was made by Hartley (1928). He suggested that "information" arises from selection of symbols or words from a given vocabulary, and introduced an uncertainty measure as a logarithm of the number outcomes of a statistical experiment. C. Shannon linked this measure to the logarithm of probability at minus sign, which, finally, became a background of entropy determination. He executed the first integrated 
mathematical attempt connected with a new concept of the amount of information and laid the foundation for the new science to be named an information theory (Shannon, 1948). Wiener's mathematical contributions have had a particular influence on the background of information theory in parallel.

The goals are to try to generalize some concepts of the information theory close to the amount of information, to improve some entropy measures throughout a prove of the uniqueness and evaluation according to Kullbach methodology, and to create the appropriate model and software for the forecast of information measures.

Besides, the information theory was accociated with the self-organization paradigm in the H. Haken fundamental work (Haken, 1988). The reduction of entropy as a result of creation of new forms itself of the system can be challenged with self-organization properties. In other contributions, the authors Gardner (1988), Buhmann et al. (1989) consider the information content obtained by calculating the entropy in neural networks when the pattern states diminished by the information deficit due to recall errors. The entropy for neuron or synapse changes and the information amount changes, too.

In this paper, the amount of information as the main evaluation measure of transmitted information in the information theory is analyzed. In the introduction and Section 2, the conceptions of the information measure relevant to the statistical physics and a short survay are considered. Section 3 is devoted to the evaluation of information by the additive rule and to an additional background of the entropy measure binding it to the evaluation of the amount of information. In Section 4, the modeling experiment by ANN and the results of the computation are discussed.

\section{Conceptions of the Information Measure Relevant to Physics}

Any of systems are defined by an ensamble of microstates. The microstates have a statistical nature and are characterized by the probabilistic laws if they are known. A statistical conception of microstates is linked with uncertainty especially if it is concomitant with the definition of information. If a new message about future microstates has been received, the uncertainty decreases, which means getting some information. That is, information is a decrease in uncertainty. Thus, information is expressed by the substraction

$$
I=S_{\text {init }}-S_{\text {final }}
$$

where $S_{\text {init }}$ serves as the measure of initial uncertainty, and $S_{\text {final }}$ is that of the final uncertainty. $I$ is the so called restricted information because it represents the reduction in the uncertainty of possible system microstates (Brillouin, 1962).

The uncertainty of microstates is characterized in the best manner by the entropy. The connection between entropy and information was first confirmed by Maxwell, who realized that a device capable of perceiving the microstates of a system could select highenergy particles and therefore violate the second law of thermodynamics. However, it is necessary to note that the entropy could not be an equivalent to information. 
In analyzing the microstate conception, it is better to retrieve the Boltzmann entropy and the maximum law in the gas medium. The entropy is expressed as

$$
S=-k \sum_{i=1}^{R} f_{i} \log f_{i}
$$

where $f_{i}$ is a probability of the $i$ th microstate, $k$ is the Boltzmann constant, $R$ is the number of microstates. Under the equiprobability law of all gas molecules, i.e., as $f_{i}=$ $1 / R$, the entropy acquires a simple form

$$
S=k \log R
$$

Here, if the constant $k$ has a real physical sense, it defines the choise of a unit of measure, according Shannon (1948).

Next, if the gas molecule expansion increases, the macrostate of gas medium becomes more symmetrical and at the final state, system reaches the maximum entropy. It is also wellknown Boltzmann the H-theorem that, if $S_{\text {init }}$ is zero, according to (1) $I$ always tends to $I \geqslant 0$ decrease.

Let the system is presented as a complex experiment consisting two stages $\alpha$ and $\beta$. The stage $\alpha$ is the preliminar measure or collection of knowledge, before carrying out the main experiment, changes the situation in the stage $\beta$. In this case, the conditional entropy $H_{\alpha}(\beta)$ under carrying out $\alpha$ will be less (correctly no more) than the entropy $H(\beta)$. If $\beta$ is independent of $\alpha$, then $H_{\alpha}(\beta)=H(\beta)$. If $\alpha$ completely defines the $\beta$ result, then the entropy of $\beta$ decreases to zero, $H_{\alpha}(\beta)=0$.

Therefore the entropy $H(\beta)$ can be used for the definition of information by

$$
H(\alpha, \beta)=H(\beta)-H_{\alpha}(\beta) .
$$

This is a possibility to define the amount of information in a cipher form.

To define the average information in (4) the entropy of all states was used

$$
H(\beta)=-\sum_{i=1}^{R} p_{i}\left(A_{i}\right) \log p_{i}\left(A_{i}\right)
$$

where $p_{i}\left(A_{i}\right)$ is a probability of the state $A_{i}$ of $\beta$ experiment. Here, to compare with (2) in formal $k$ equal to 1 . The $H_{\alpha}(\beta)$ in (4) is the entropy expressed by conditional probabilities. The $H(\beta)$ measures by bits per symbol, which is equivalent to a state in the binary target.

Another conception of information measure can be expressed through possible pathways of dissipation. The dissipation can be interpreted as the heat produced within the system. For closed systems at the steady state, the dissipation must be exactly compensated by the heat exported to the environment. Thus, an entropy defined over the possible steady states reaches the maximum if to assume $a$ priori an equal probability of their 
occuring. Of course, the number of potential steady states (or more generally, pathways of dissipation) could become very large. This conception of information measure may be called as an unrestricted information paradigm.

A third conception of the evaluation of information is based on self-organization processes relevant to the self-organization or ordered structures that reduce the entropy in creating the form itself. Here, the initial state of microstates out of which the ordered set is sellected plays an important role. In other words, the initial entropy is calculated over a relevant initial set of microstates before the particular macrostate is prepared. The information is expressed as the difference between the initial entropy and the final entropy over the final set of microstates.

The various distinctions between microscopic and macroscopic uncertainty of system state transitions and between these and the homogeneity or inhomogeneity of the states, play a fundamental role in the development of the information theory.

\section{Evaluation of Information by Additive Rule}

\subsection{Uniqueness of the Entropy Measure}

We have adopted a logarithmic form in formal use of Boltzmann entropy and additional presumptions and leading to a simple form of entropy (3). Allowing a few specified requirements as the constraints, we prove that the logarithmic form is the unique function satisfying the constraints as follows:

1. In a finite complete probability case, the associated entropy function must take the largest value when all events are equiprobable.

2. In a joint finite complete case, the entropy satisfies the information evaluation form (4).

3. The entropy function is continuous, nonnegative, it grows with an increase in the number of events, and does not change after transposing the events in the event succession.

The prove that the uniqueness of the entropy function in the equiprobable case is simple and it can be found in Shannon's (1948), Khinchin's (1970) and, for less restrictive conditions, in Fadiev's (1956) contributions.

Hereby, we consider the case of the proof of an unique solution where all the events are not equiprobable and the probabilities may be rational numbers.

Let $a$ be a general denominator of different rational $p_{k}$ as follows

$$
p_{k}=a_{k} / a ; \quad \sum_{k=1}^{R} a_{k}=a, \quad a_{k}>0 .
$$

Further, we rename the experiment $\alpha$ as $X$ and $\beta$ as $Y$ for the general case of events, considering a probability $Y$ depending on $X$. Let $\mathbf{Z}=\left(z_{1}, z_{2}, \ldots, z_{\alpha}\right)$ consists of $m$ equally like events. For convenience, we devide $\mathbf{Z}$ events into groups containing 
$a_{1}, a_{2}, \ldots, a_{k}$ events. This scheme refers to the $Y$. All events of $X$ are devided into $k$ groups. The events in gruops can occur with an equal probability. Then

$$
H\left(1 / a_{k}, 1 / a_{k}, \ldots, 1 / a_{k}\right)=\lambda \log a_{k} .
$$

Here $a_{k}$ is the number of events in the $k$ th group and the $\lambda$ is a positive constant.

The conditional entropy

$$
H(Y \mid X)=\sum_{k=1}^{R} p_{k} H\left(1 / a_{k}, 1 / a_{k}, \ldots, 1 / a_{k}\right)=\lambda \sum_{k=1}^{R} p_{k} \log p_{k}+\lambda \log m .
$$

The total entropy in a two-dimensional case $(X, Y)$ for $\mathbf{Z}$ events will be as follows

$$
H(\mathbf{Z})=H(X, Y)=\lambda \log m .
$$

According to the additivity requirement of (8) and (9)

$$
H(X)=H(X, Y)-H(Y \mid X)=-\lambda \sum_{k=1}^{R} p_{k} \log p_{k} .
$$

Thus, the uniqueness of solution is valid when $p_{k}$ are rational numbers.

\subsection{Evaluation of the Information Amount}

The amount of information as the main category of the information theory depends on the properties of functions and their additivity associated with the number of realizations of events. Now we describe, with some generalizations, the Kullbach (1959) manner of evaluating information.

Let a message consist of $N_{1}$ letters, $N_{2}$ ciphers, and $N_{3}$ mathematical and grammatical signs with the common number of events

$$
N=\sum_{i=1}^{3} N_{i}
$$

Then the number of possible realizations will be

$$
R=N ! / N_{i} ! N_{2} ! N_{3} !
$$

According to (3) or (9)

$$
I=\lambda \log R=\lambda\left[\log N !-\log N_{1}-\log N_{2}-\log N_{3}\right] .
$$

Using the Stirling's approximation

$$
\log Q ! \cong Q(\log Q-1),
$$


where $Q$ is the number of realizations in general case.

After some mathematical manipulations, we get that

$$
I \cong-\lambda\left[N_{1} \log N_{1} / N+N_{2} \log N_{2} / N+N_{3} \log N_{3} / N\right]
$$

or

$$
i=I / N \cong-\lambda\left[N_{1} / N \log N_{1} / N+N_{2} / N \log N_{2} / N+N_{3} / N \log N_{3} / N\right] .
$$

Assume that at a large number $N$ the relations $N_{j}(j=1,2,3)$ become as probabilities $p_{j}$. Then we get the Shannon formula

$$
i=-\lambda \sum_{j=1}^{N} p_{j} \log p_{j}
$$

Taking in to account that $i_{j}=-\log p_{j}$ as information is dependent on the event $j$ in the a priori measure and $i_{j}^{\prime}=-\log p_{j}^{\prime}$ in the a posteriori measure, the difference

$$
i_{j}^{\prime}-i_{j}=\lambda \log p_{j} / p_{j}^{\prime}
$$

will present an information gain. Given the average of $N$ events, we obtain the KullbachLeibler measure (Khinchin and Gnedenko, 1970) generalized and presented as follows

$$
i=-\lambda \sum_{j=1}^{N} p_{j} \log p_{j} / p_{j}^{\prime}
$$

where $i \geqslant 0, \lambda \geqslant 0$ and $\sum_{j=1}^{N} p_{j}=\sum_{j=1}^{N} p_{j}^{\prime}=1$.

In addition, if $p_{j}$ and $p_{j}^{\prime}$ are the probabilities achieving the goal, then

$$
E=\lambda \log p_{j} / p_{j}^{\prime}
$$

will define the information value. And if $p_{j} \approx 1$, then $E=-\lambda \log p_{j}^{\prime}$ and the information value in the same as the information amount.

\section{Modeling Experiment by ANN}

\subsection{Preprocessing to Modeling Experiment}

All messages can be very long and repeated with a certain frequency under a different stimulus. The resulting response vector could be $n$-dimensional and may be denoted as $\mathbf{r}$ with the components $r_{t}$. These data could be compressed, expressing the responses 
in terms of their principal components (Oja, 1997; Hyvärinen and Oja, 1996). These components of $r_{t}$ are based on eigenvalues of the covariance matrix

$$
C_{t t^{\prime}}=\left\langle\left(r_{t}-\left\langle r_{t}\right\rangle\right)\left(r_{t^{\prime}}-\left\langle r_{t^{\prime}}\right\rangle\right)\right\rangle,
$$

where $\langle\cdot\rangle$ is the sign of the average of deviations or expectations $r_{t}$ and $r_{t^{\prime}}$ of the appropriate components of the response vector.

Usually the principal components are ordered according to the magnitudes of their eigenvalues. This transformation is the key of compression of the data supported by the higher principal components with the least amount of variance. The compression limits the space of search in which the characteristics of the message response are placed. Further, the principal components are redenoted by the same $r_{j}(j=1,2, \ldots, n)$ of the vector r. In our modeling experiment, a source of messages transmits a signal $\mathbf{r} \in R$ in response to a stimulus $s \in S$, where $R$ is an $n$-dimensional space and $S$ is a set of stimuli, respectively. Our goal is to define the information amount in response to the stimulus. Now the conditional probability $P(s \mid \mathbf{r})$ of stimulus $s$, given the response $\mathbf{r}$, becomes the main object in the definition of transmitted information expressed by fitting measure (19) as follows

$$
I(S, R)=\sum_{s=1}^{S}\langle P(s \mid \mathbf{r}) \log P(s \mid \mathbf{r}) / P(s)\rangle_{\mathbf{r}}
$$

where the average is taken over the message response distribution, and $P(s)=\langle P(s \mid \mathbf{r})\rangle_{\mathbf{r}}$ is the unconditional probability of stimulus s. To estimate $I(S, R)$, we must first estimate $P(s \mid \mathbf{r})$ corresponding to the message data. The quality criterion used is a standard statistical one based on a $\log$-likelihood measure. We suppose that $K_{s}(\mathbf{r})$ is the estimate of $P(s \mid \mathbf{r})$ provided by the ANN model bellow. It is necessary to minimize the cost function

$$
e=-\sum_{\mu s} T_{s}^{\mu} \log K_{S}\left(\mathbf{r}_{\mu}\right)
$$

The summation index $\mu$ denotes the message data examples used to make the fit it, and the target function $T_{s}^{\mu}$ is equal to 1 if the response example $\mu$ was evoked by stimulus $s$, and 0, otherwise. Expression (23) is the Kullbach-Leibler measure or (19) after reducing it to a binary target.

\subsection{Building of ANN}

To realize an appropriate fitting procedure evaluating the transmitted information, the artificial neural network methodology was used. To this goal one may implement different kinds of ANN architecture (Rosenblatt's perceptron, one-layered Hopfield's network, Kosko's bi-directional net, recurrent networks, radial basis, and others). We have chosen a multi-layered learning algorithm with backpropagation procedure. For this kind of ANN, the main characteristic parameters are the number of hidden units. The fitting procedure 
involves other adjusting parameters, such as the connection weights among neurons so as to minimize the cost function. Two sets of messages were used to train and to test the network.

The scheme of ANN, based on a layered neural network with a single hidden layer, is presented in Fig. 1. It consists of an input (buffer) layer, one hidden layer with bipolar sigmoidal activation functions, and an output layer, where units work is based on the normalized exponential transit functions. There are full connections from one layer to another and no connections inside a layer. The ANN possesses one bias unit for a hidden layer and another for the output one. The input data are certain (from one or more) principal components $r_{j}$ of message response $\mathbf{r}$. An output layer has as many units as stimulus classes $S$ are updated.

In contrast to the most commonly used ANN scheme in the output units the normalized exponential activation functions of weighted sums of hidden unit activation were used. This joint log-likelihood cost function (23) causes the output to give a direct estimate of the conditional probabilities $P(s \mid \mathbf{r})$.

When the input $\mathbf{r}$ is introduced into the network, the hidden unit actvation $i$

$$
H_{i}(\mathbf{r})=\tanh \left(\sum_{j=1}^{n} W_{i j} r_{j}+b_{i}\right)
$$

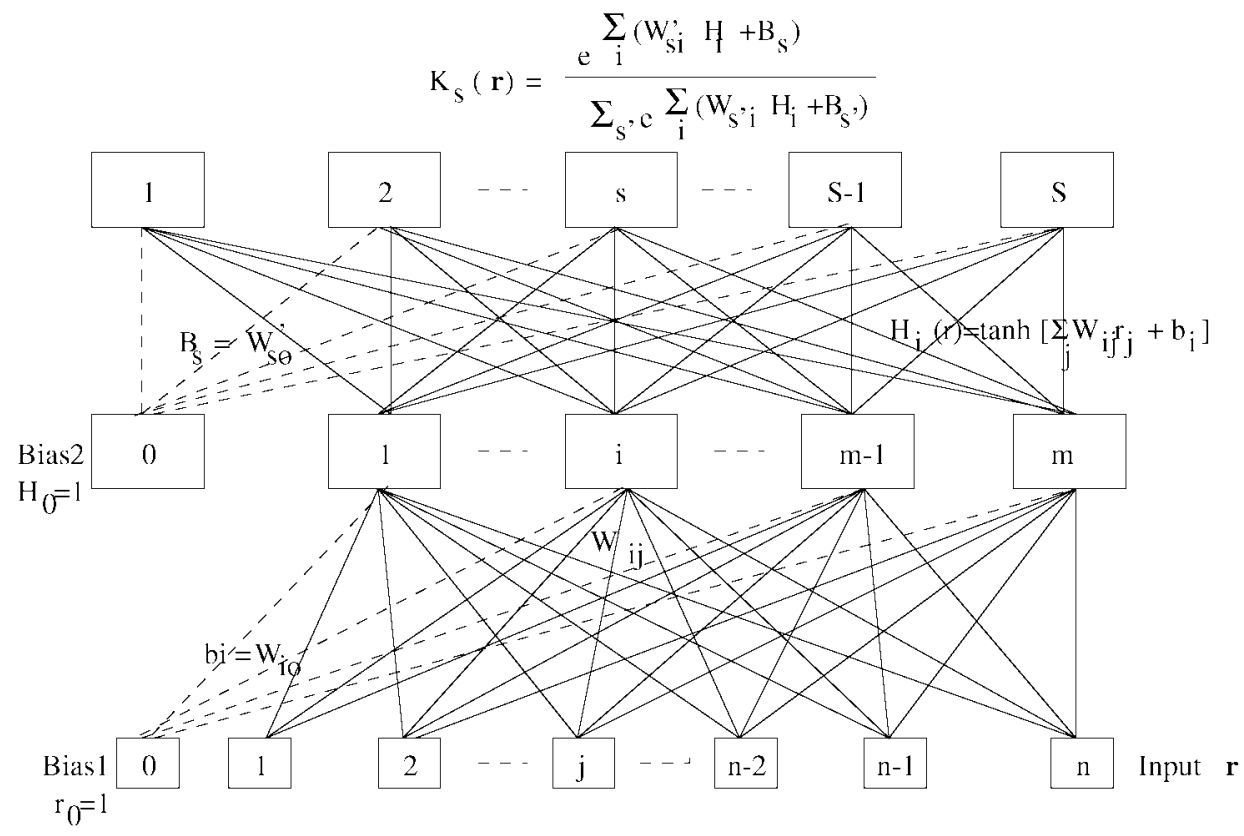

Fig. 1. Neural network scheme to decode information of messages. The scheme based on bipolar sigmoid and normalized exponential functions, and involves input layer with $n$, hidden layer with $m$, and output layer with $S$ units including two bias units. 
and the output activation is

$$
K_{s}(\mathbf{r})=\frac{\exp \left[\sum_{i}\left(W^{\prime}{ }_{s i} H_{i}+B_{s}\right)\right]}{\sum_{s^{\prime}} \exp \left[\sum_{i}\left(W_{s^{\prime} i} H_{i}+B_{s^{\prime}}\right)\right]} .
$$

The proper parameters (weight and bias) are searched by an iterative backpropagation algorithm. The learning algorithm includes the following steps:

1. According to the message response vector for the example $\mu$ of the learning set $\mathbf{r}_{\mu} \in R$ and the values randomly given weights $W_{i j}, H_{i}\left(\mathbf{r}_{\mu}\right)$ are calculated. By analogy, $W_{s i}$ and $K_{s}\left(\mathbf{r}_{\mu}\right)$ are defined. This step constitutes the feed-forward procedure.

2. The feedback procedure is realized under the gradient formula

$$
\Delta W_{s i}=\eta_{1} \sum_{\mu}\left[T_{s}^{\mu}-K_{s}\left(\mathbf{r}_{\mu}\right)\right] H_{i}\left(\mathbf{r}_{\mu}\right)
$$

where $\Delta W_{s i}^{\prime}=W_{s i}^{\prime}$ new $-W_{s i}^{\prime}$ old,$\eta_{1}$ is a learning rate.

The changes of weights in the hidden layer as updated according to

$$
\Delta W_{i j}=\eta_{2}\left\{\sum\left[T_{s}^{\mu}-K_{s}\left(\mathbf{r}_{\mu}\right)\right] W_{s i}\right\}\left(1-H_{i}^{2}\left(\mathbf{r}_{\mu}\right) r_{j}^{\mu},\right.
$$

where $1-H_{i}^{2}\left(\mathbf{r}_{\mu}\right)$ is a derivative of the sigmoidal function (24), $\eta_{2}$ is the second learning rate in the range $0 \leqslant \eta_{2} \leqslant 1$. Afterwards the new weight values are calculated according to $W_{s i}^{\text {new }}=W_{s i}^{\text {old }}+\Delta W_{s i}$ and $W_{s i}^{\prime}$ new $=W_{s i}^{\prime}$ old $+\Delta W_{s i}^{\prime}$. Then the feed-forward procedure is repeated and the iterative search is continued looking for the minimum error or testing the convergence condition $\mathrm{e}_{\mathrm{min}}$, if such was foreseen.

Sometimes the overfitting, when the training error starts to rise, can be expected here. The test set is stopped, i.e., when the test error reaches the minimum.

\subsection{Modeling Experiment}

The feedback neural network learning algorithm has been implemented in "Neuron 7.0" software. The programming realization was based on an experience expressed in the recent contributions (Garliauskas and Shaw, 1997; Garliauskas, 2003).

For experimental modeling according to our approach, we have chosen an unusual but interesting field of application. This field is related to the analysis of genetic information. It is known that the genetic code is formed by sequences of nucleotides (Adenine (A), Cytosine (C), Guanine (G), and Urasine (U)). It is necessary to emphasize that the degree of biological sequence conservation is given in bits of information. The sequence conservation can be represented by graph of an aligned set of bindings among nucleotides (protein or macromolecular complex). In Schneider and Stephens (1990), and Gorodkin et al.) (1997) contributions, where was proposed a sequence logo display of the frequencies of the repeated appearence of nucleotides, as the relative hight of their letters of 
expressing the summary hight, as the amount of information in bits. The example of such a logo is shown in Fig. 2.

It is built based on combinations of nucleotides from the table as follows:

CCAGAGGCCCAACUGGUAAACGGGC
CCGUAAGCUCAACGGGAUAAUGAGC
CCGGAAGCCGAACGGGAAAACCGGC
CCCCAAGCGC-AGGGGAGAAAGCGC
CCGAACGCCAUACGGGAGAA-UGGC
CCGUUUUCAGAUCGGGAAAAACUGA
CCGUUACUCCAUCGGGAUAAAGGAG
CCGUAAGAGGAACGGGAUAAACCUC
CCGCUAGGAGGGCGGGAUAUACUCC
CCGAGUGCCGAGCGGGAUAUACGGC
CCGCAACUCGAACGGGAUAAACGAG
CCGCCACUCGCACGGGAUAAACGAG

The a priori probabilities $p(A), p(C), p(G)$, and $p(U)$ were taken as equal to 0.25 . The information content in the logo graph for structure alignments including gaps among nucleotides and mutual information for RNA is discussed in (Gorodkin et al., 1997). Regarding this contribution, the basepair probabilities, which control a relative scaling between the sequence structure and mutual structure of information, were given $p(A U)=$ $0.8, p(C G)=0.9$, and $p(G U)=1.0$.

The main task in the genetic sequence analysis is the RNA structure prediction. Based on the data expressed by cipher information in bits instead letters in logo display (Fig. 2) we tried to learn and forecast the structure of nucleotide combination (NC) in the future. The initial graph (a) of NC and the predicted curve (b) of information amount $i$, and the estimation of cost function versus learning cycles are presented in Fig. 3 and Fig. 4

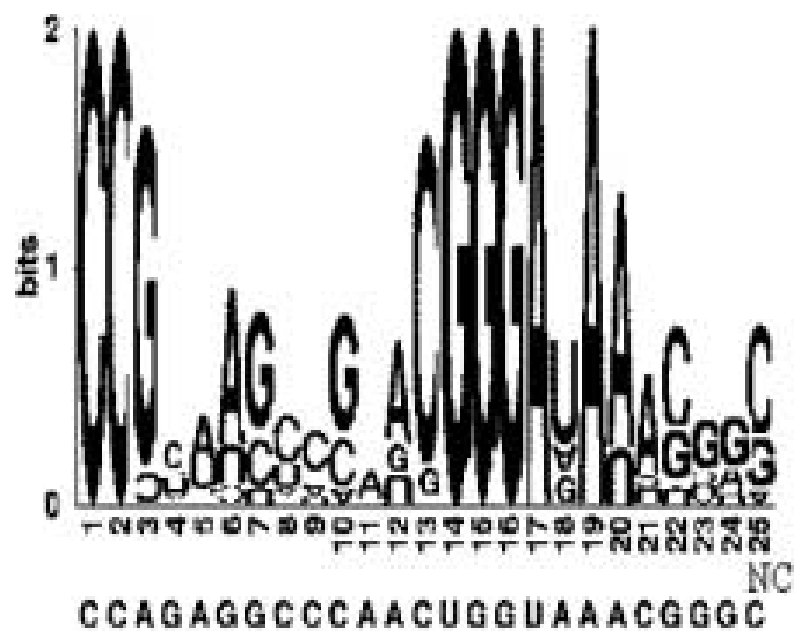

Fig. 2. The example of a sequence logo graph built by Schneider's method (Schneider and Stephens, 1990). 


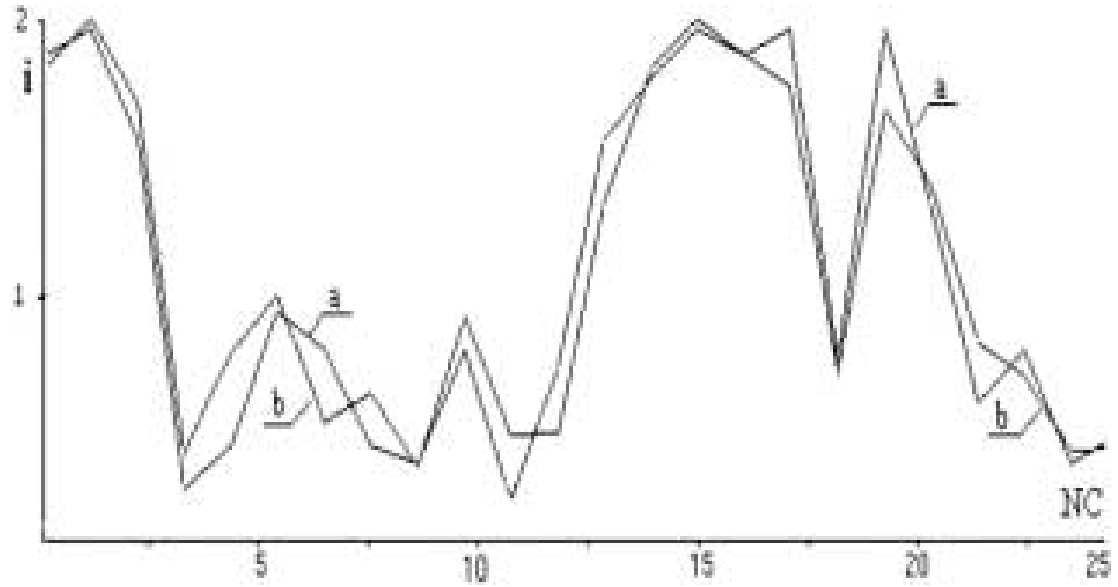

Fig. 3. The results of the forecast for biological information. (a) The initial graph of NC and (b) the predicted curve of the information amount in bits are presented.

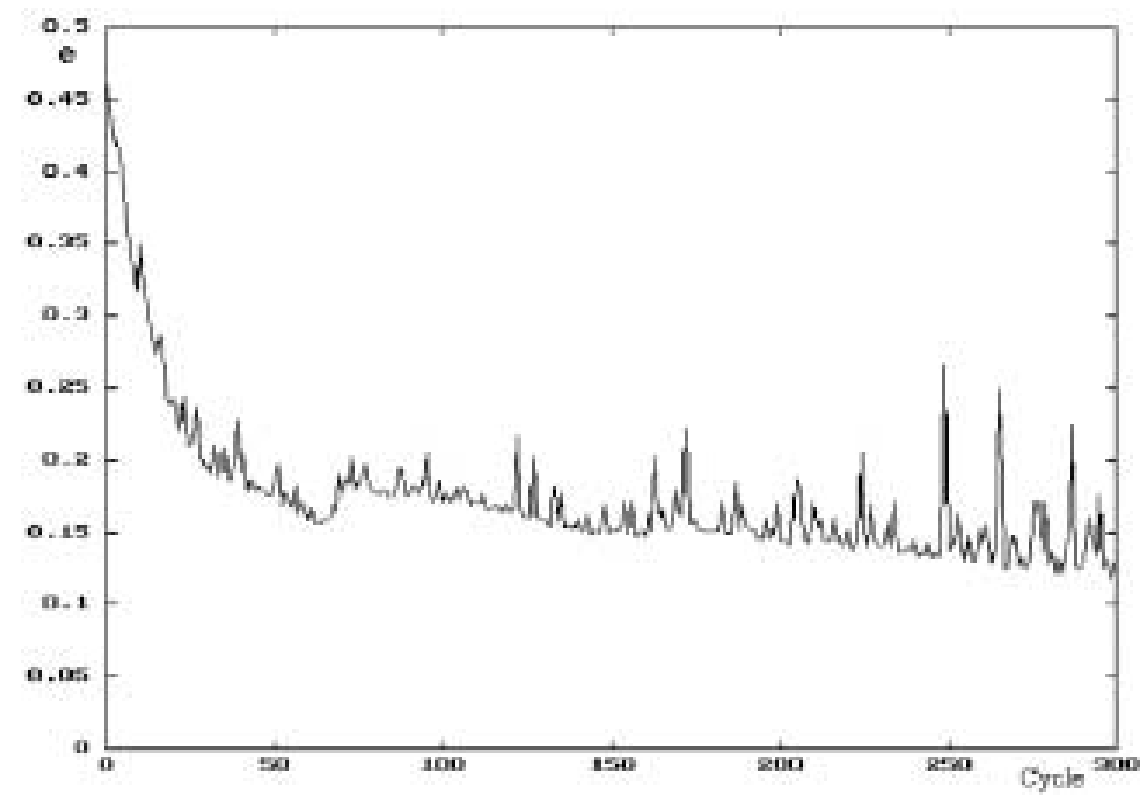

Fig. 4. The cost function versus learning cycles.

respectively. This simple illustration example shows that the forecast of the amount of information by ANN created upon the submitted method yields, in comparison, satisfying results and can be applied, we hope, in different practical areas. 


\section{Conclusions}

1. Since the evaluation of transmitted information in the information theory is based on the probabilistic event methodology, three conceptions of the definition of information, including the self-organization, have been considered.

2. The proof of uniqueness of the entropy measure was interpreted as a new modification of proving when all events are not equibrobable and the probabilities are rational numbers under a few specified requirements.

3. The use of the artificial neural networks approach based upon the conditional probability method for measuring the transmitted information and application of the log-likelihood cost function criterion allowed us to construct the model, the rationality of which is illustrated by evaluating the biological genetics information.

\section{References}

Boltzmann, L. (1868). Studien über das Gleichgewicht der lebendige Kraft zwischen bewegten materiellen Punkten, Akademie der Wissenschaften in Wien. Mathematisch - Naturwissenschaftliche Klasse IIa, 58, 517-560.

Brillouin, L. (1962). Science and Information Theory. Academic Press, NY

Buhmann, J., R. Divko and K. Schulter (1989). Associative memory with high information content. Physical Review A, 39(5), 2689-2692.

Fadiev, D.K. (1956). To concept entropy in final probability scheme. Advanc. Math. Science, 11(1), 227-234 (in Russian).

Gardner, E. (1988). The space of interactions in neural network models. J. Phys. A: Math. Gen., 21, 257-270.

Garliauskas, A. (2003). Neural network computation of forecast in finance with chaotic aspects. International Journal of Computational and Numerical Analysis and Applications, 3(3), 263-275.

Garliauskas, A., and M. Shaw (1997). Neural network chaos theory and applications in finance. Computers in Finance and Economics, Proc. of PASE'97, 45-54.

Gorodkin, J., L.J. Heyer, S. Brunak and C.D. Stormo (1997). Displaying the information contents of structural RNA alignments: the structure logos. Comput. Appl. Biosci., 13(6), 583-586.

Haken, H. (1988). Information and Self-Organization, Berlin.

Hartley, R.V.L. (1928). Transmission of information. Bell System Tech. J., 7, 535-564.

Hyvärinen, A., and E. Oja (1996). Simple neuron models for independent component analysis. Int. Journal of Neural Systems, 7, 671-687.

Khinchin, V.I., and B.V. Gnedenko (1970). The Elementary Introduction to the Theory of Probabilities, Science Press, Moscow (in Russian).

Kullbach, S. (1959). Information Theory and Statistics, Willey, NY, 197-200.

Oja, E. (1997). The nonlinear PCA learning rule in independent component analysis. Neurocomputing, 17, 25-45.

Schneider, T.D., and R.M. Stephens (1990). Sequence logos: a new way to display consensus sequences. Nucleic Acids Research, 18(20), 6097-6100.

Shannon, C.E. (1948). A mathematical theory of communication. Bell System Tech. J., 27, 379-423, 623-656.

Shannon, C.E., and W. Weaver (1949). The Mathematical Theory of Communication, Univ. of Illinois Press, Urbana. 
A. Garliauskas is a habilitation doctor of technical sciences, professor of Data Analysis Department of Institute of Mathematics and Informatics, a member of the Council of Lithuanian Science Society. He received $\mathrm{PhD}$ and habilitation doctor in the field of research operation and system analysis from Lithuanian Science Council 1994. His research work is the modeling complex systems and neural networks. He has published over 175 scientific articles, 5 monographs. Last 15 years he deals with the problems of the nonlinear neural networks, chaos and fuzziness processes in order to improve the methodology of signal and image recognition. 
Perduodamos informacijos iqvertinimo koncepcijos ir modeliavimas dirbtiniais neurotinklais

\author{
Algis GARLIAUSKAS \\ Straipsnyje analizuojami ir tikslinami pagrindiniai informacijos teorijos matai ir informacijos \\ kiekio įverčiai. Nagrinejjamos trys teorijos koncepcijos mikrobūsenu, disipacijos ir saviorganizaci- \\ jos lygmenyse. Irodytas entropijos funkcijos vienatiškumas aprěžtos informacijos atvejui. Remian- \\ tis dirbtinio neurotinklo modeliu atliktas eksperimentinis įvertinimas biologinès informacijos sri- \\ tyje.
}

